\title{
Cuantificación del riesgo de enfermedad cardiovascular, según el score Framingham, en el personal militar durante el año 2015
}

\section{Quantification of cardiovascular disease risk, according to the Framingham score, in military personnel during $\mathbf{2 0 1 5}$}

\author{
Alberth Muñoz ${ }^{1 *}$ y Germán Muñoz ${ }^{2}$ \\ ${ }^{1}$ Universidad Nacional de Loja, Facultad de Salud Humana, Loja, Ecuador. \\ ${ }^{2}$ Fuerzas Armadas del Ecuador, Grupo de Fuerzas Especiales № 26, “Cenepa”, Los Ríos, Ecuador. \\ * Autor de correspondencia: albmu93@gmail.com
}

DOI: https://doi.org/10.26807/remcb.v39i1.560

Recibido 20-11-2017; Aceptado 23-03-2018

\begin{abstract}
RESUMEN.- Las enfermedades cardiovasculares son la principal causa de muerte en el mundo y más de las tres cuartas partes de ellas se presentan en países de ingresos bajos y medios. Los factores de riesgo se definen como características biológicas que aumentan la probabilidad de padecer una enfermedad. En la presente investigación se demuestra el riesgo cardiovascular total, los principales factores de riesgo y el riesgo, según la edad, en la población militar. Este es un estudio cuantitativo, descriptivo y transversal, que analiza una muestra de 80 militares del Batallón de Selva No 62 "Zamora" y que usa como referencia el test de riesgo de Framingham. Los resultados mostraron que el riesgo absoluto de enfermedad cardiovascular es bajo con el 60\%. Entre los principales factores de riesgo se encuentran HDL bajo con el 80\%; seguido de sobrepeso, con el 46,25\%. En la población de entre 30 a 39 años el riesgo es bajo con el 81,63\%; en la población de entre 40 a 49 años el riesgo es moderado con el 60\%; y, en la población de 50 años o más, el riesgo es moderado con el 100\%. Se concluye, así, que el riesgo cardiovascular en la población militar se ubica entre bajo y moderado, siendo sus principales factores de riesgo las alteraciones de lípidos y el sobrepeso.
\end{abstract}

PALABRAS CLAVES: atención primaria, epidemiología, factor de riesgo, Framingham, riesgo cardiovascular.

\begin{abstract}
Cardiovascular diseases are the world's leading cause of death world, with more than three quarters of cases occurring in low- and middle-income countries. Risk factors are defined as biological characteristics that increase the probability of suffering from a disease. In this investigation, we demonstrate overall cardiovascular risk, main risk factors and risk according to age in the military population. The study is quantitative, descriptive and cross-sectional, using a sample of 80 soldiers from the Jungle Battalion No. 62 "Zamora." The Framingham risk test is used as a reference. The absolute result obtained is that the absolute risk of cardiovascular disease is low, at $60 \%$. Among the main risk factors are low HDL, at $80 \%$, followed by excess weight, at $46.25 \%$. In the population between 30 to 39 years old, risk is low, at $81.63 \%$; for the population between 40 to 49 years old, risk is moderate, at $60 \%$; and, in the population aged 50 years and above, risk is moderate, at $100 \%$. It is concluded that cardiovascular risk in the military population ranges between low and moderate, with the primary risk factors being alterations of lipids and excess weight.
\end{abstract}

KEYWORDS: cardiovascular risk, epidemiology, Framingham, primary care, risk factor.

\section{INTRODUCCIÓN}

Los factores de riesgo cardiovasculares se definen como una característica biológica o hábito de vida que aumenta la probabilidad o el riesgo de padecer una enfermedad cardiovascular mortal en aquellos individuos que lo presentan (Lobos y Castellanos 2006). Dentro de los cuales, se pueden identificar: tabaquismo, hipertensión arterial, hipercolesterolemia, género masculino, obesidad, alimentación inadecuada, falta de ejercicio físico, etnia, diabetes mellitus y presencia de antecedentes familiares (Lobos y Brotons 2011; Amariles et al. 2004; O'Donnell y Elousa 2008). Las enfermedades cardiovasculares son la principal causa de muerte en todo el mundo, repre- 
sentando aproximadamente un $31 \%$ de las mismas, de las cuales más de las tres cuartas partes se presentan en países de ingresos bajos y medios (OMS 2015).

Se han desarrollado varios modelos para valorar el riesgo cardiovascular. El modelo de Framingham es ampliamente usado en Norteamérica, por la similitud de la población. Por otro lado, la guía europea de prevención cardiovascular propone el modelo SCORE (Systematic Coronary Risk Evaluation). Mientras el modelo Framingham estima el riesgo a 10 años de infarto agudo de miocardio y muerte coronaria, el modelo SCORE, además de las enfermedades coronarias, valora cualquier otro proceso cardiovascular aterotrombótico, teniendo en cuenta solo el riesgo de complicaciones mortales (Backer et al. 2003).

Al realizar un análisis entre los resultados de varios estudios que comparan el modelo SCORE y el modelo Framingham, se encontró que 4 estudios españoles concluyen que el modelo SCORE clasifica como de riesgo alto a un número significativamente mayor de participantes (Jaume et al. 2003; Mostaza et al. 2005; González et al. 2006, Cabrera et al. 2009). En comparación a un solo estudio español que demuestra lo contrario (Galán et al. 2004). Además, en un estudio realizado en México, se concluye que es más adecuado el uso del modelo Framingham, debido a que el modelo SCORE subestima el riesgo (Alcocer et al. 2011). En estudios realizados en Chile y Colombia, los mismos que evalúan el uso del modelo Framingham sin relacionarlo al modelo SCORE, se sugiere que la escala Framingham debería usarse con precaución en la población sudamericana, ya que presentan diferentes características poblacionales en comparación con la población en la cual se basó el modelo (Icaza et al. 2009; Muñoz et al. 2014).

En la década de 1950, se iniciaron estudios epidemiológicos que buscaban determinar las causas de las enfermedades cardiovasculares. En Boston (Estados Unidos), en el año 1948, se inicia el Framingham Heart Study con una primera cohorte de 5209 habitantes sanos, de entre 30 a 60 años de edad, a quienes se les realizó exámenes bianuales. En el año 1971, se agregaron a 5124 hijos e hijas (y sus cónyuges) de la primera cohorte para continuar con el Offspring Study (estudio de la descendencia). En el año 2002, se 4095 participantes para el estudio Third Generation (tercera generación) (Dawber et al. 1951; Dawber et al. 1957; Splansky et al. 2007). Luego de los estudios, se determinó que los primeros factores de riesgo identificados eran el colesterol elevado y la presión arterial alta (NHI 1957).

Para la valoración de los factores de riesgo cardiovasculares se usa la información obtenida a partir de los grandes estudios prospectivos expresados en el score de Framingham, organizando a los mismos en riesgo bajo (menos de $5 \%$ ), riesgo moderado (de 5 a $9.9 \%$ ), riesgo alto (de 10 a $14.9 \%$ ) y riesgo muy alto (mayor de 15\%) (Álvarez 2001; Armario et al. 2005; D’Agostino et al. 2008).

Las enfermedades cardiovasculares se encuentran dentro de las enfermedades crónicas no transmisibles, cuya prevalencia e incidencia tiende al alza debido al cambio de hábitos en la población en general. La responsable de su desarrollo y sus manifestaciones es la enfermedad ateroesclerótica, con sus cuatro principales consecuencias orgánicas: enfermedad arterial coronaria, enfermedad cerebrovascular, enfermedad arterial periférica y aneurismas ateroescleróticos (Vega et al. 2011).

Debido al constante estrés físico y mental al que está expuesta la población militar, se considera necesario conocer la realidad cardiovascular de los mismos. Es fundamental la detección precoz y tratamiento temprano para poder evitar riesgos en el ámbito laboral en el que se desenvuelven, además de disminuir la morbimortalidad de la población en estudio. A través de la presente investigación se pretende demostrar el riesgo cardiovascular total, los principales factores de riesgo y el riesgo según la edad en la población militar.

\section{MATERIALES Y MÉTODOS}

El estudio fue de tipo cuantitativo, descriptivo y transversal. Se utilizó como fuente de recolección de información a las fichas médicas anuales correspondientes al año 2015 del personal militar del Batallón de Selva $\mathrm{N}^{\mathrm{o}} 62$ "Zamora", las mismas que fueron realizadas en el Hospital Militar de Brigada $N^{\circ} 7$ "Loja". La ficha médica anual es un requisito obligatorio de medicina preventiva que tiene el Comando del Ejército como política de seguimiento y control médico del personal militar. Parte de la ficha médica anual corresponde a: chequeo médico, psicológico y odontológico, exámenes de laboratorio, examen de agudeza visual, audiometría, control de signos vitales y antropometría.

Se investigaron a los profesionales militares del Batallón de Selva No 62 "Zamora", ubicado en la Provincia de Zamora Chinchipe (Ecuador), dentro de los cuales se encuentran oficiales y voluntarios, con una población de 251 militares. La muestra está comprendida por militares que cumplen los criterios de inclusión: a) Personal militar efectivo del Batallón de Selva No 62 "Zamora"; b) edad comprendida entre los 30 y 70 años; y c) ausencia de enfermedad coronaria. Además, no se tomó en consideración a aquellos participantes cuyas fichas médicas estuviesen incompletas. 
Al finalizar el año 2015, se realizaron las fichas médicas anuales al personal militar del Batallón de Selva $\mathrm{N}^{\mathrm{o}} 62$ "Zamora", mismas que fueron realizadas en el Hospital de Brigada $N^{\circ} 7$ "Loja". Los datos antropométricos se tomaron en iguales condiciones: usando una báscula de piso SECA 874 y una cinta ergonómica SECA 201. La presión arterial fue tomada a todos los participantes con el mismo esfigmomanómetro de mercurio, con el paciente en sedestación y luego de 5 minutos de reposo. Los exámenes de laboratorio, en cambio, fueron realizados dentro de la Institución, en similares condiciones. Posteriormente, se analizó las fichas médicas anuales, verificando los criterios de inclusión y exclusión. Se encontró que, del universo a investigar, 80 individuos cumplían con los estándares previstos.

Se definió, entonces, ciertos conceptos según medicina basada en evidencia: Hipertensión arterial (presión arterial $>140 / 90$, o diagnóstico instaurado y/o uso de medicación antihipertensiva), sobrepeso (IMC > 25 a 29.9), obesidad (IMC > 30), colesterol total normal $(<200)$ colesterol alto ( $>200)$, HDL bajo $(<50 \mathrm{mg} / \mathrm{dl})$ HDL normal (> $50 \mathrm{mg} / \mathrm{dl})$ (ALAD, 2013). La información para el estudio se obtuvo gracias a la autorización previa del Comandante del Batallón de Selva No 62 "Zamora". Además, se respetó la privacidad de cada participante con previo consentimiento informado.

Se ingresó la información obtenida de las fichas médicas anuales en una matriz previamente establecida en el programa Microsoft Excel Profesional. Posteriormente, se calculó el riesgo de enfermedad cardiovascular a 10 años del personal militar del Batallón de Selva No 62 "Zamora", con la utilización del programa Cardiovascular Disease (10-year risk) and Calculator (FHS 2016).

\section{RESULTADOS}

La muestra estudiada estuvo integrada por una población netamente masculina y la mayor parte joven, siendo el $61.25 \%$ personal comprendido entre 30 y 39 años. El $78.75 \%$ de participantes se autodenominaron mestizos, seguidos por el $16.25 \%$ de nativos y $5 \%$ de la etnia negra. En cuanto a los factores de riesgo, la mayoría no presenta antecedentes patológicos personales de diabetes mellitus tipo $2(98.75 \%)$ o hipertensión arterial (100\%). El $76.25 \%$ del personal menciona que no fuma. El $46.25 \%$ presenta valores normales de presión arterial, seguida del $36.25 \%$ con valores de pre-hipertensión. Existe similitud entre el personal militar que presenta un índice de masa corporal normal $(47.50 \%)$ y sobrepeso $(46.25 \%)$. La mayoría $(58.75 \%)$, mantiene un registro normal de colesterol total. Además, se encontraron valores de colesterol HDL bajo en el $80 \%$ del personal militar

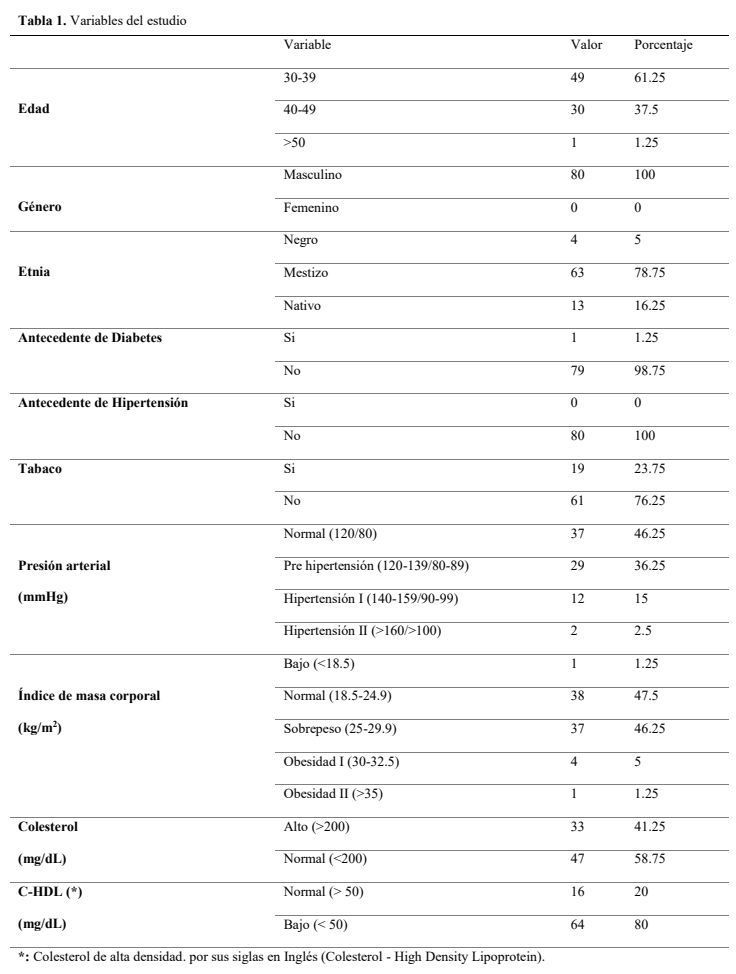

Aplicando el score de Framingham, se evidencia que el riesgo absoluto de enfermedades cardiovasculares en el personal militar del Batallón de Selva No 62 "Zamora” es bajo en el de la población (60\%); seguido por personal militar que presenta un riesgo moderado (33.75\%)

El mayor porcentaje de riesgo bajo de enfermedades cardiovasculares se da en personal de etnia negra (100\%), seguida por los mestizos (58.73\%) y los nativos (53.85\%). El riesgo alto se evidencia en el $15.38 \%$ en la población militar nativa

En cuanto a la edad, el riesgo es bajo en el $81.63 \%$ de la población comprendida entre 30 y 39 años; en cambio, en el $60 \%$ de la población de entre 40 y 49 años el riesgo es moderado; y en el 100\% de la población de 50 años en adelante el riesgo es moderado (Figura 3,).

\section{DISCUSIÓN}

En la actualidad, al ingresar a la carrera militar, es necesario cumplir con una serie de características psicológicas y físicas determinadas. Los aspirantes son evaluados previamente al ingreso a cualquier rama de las Fuerzas Armadas y, a través de controles periódicos, se busca constantemente la presencia de factores de riesgo. Por 


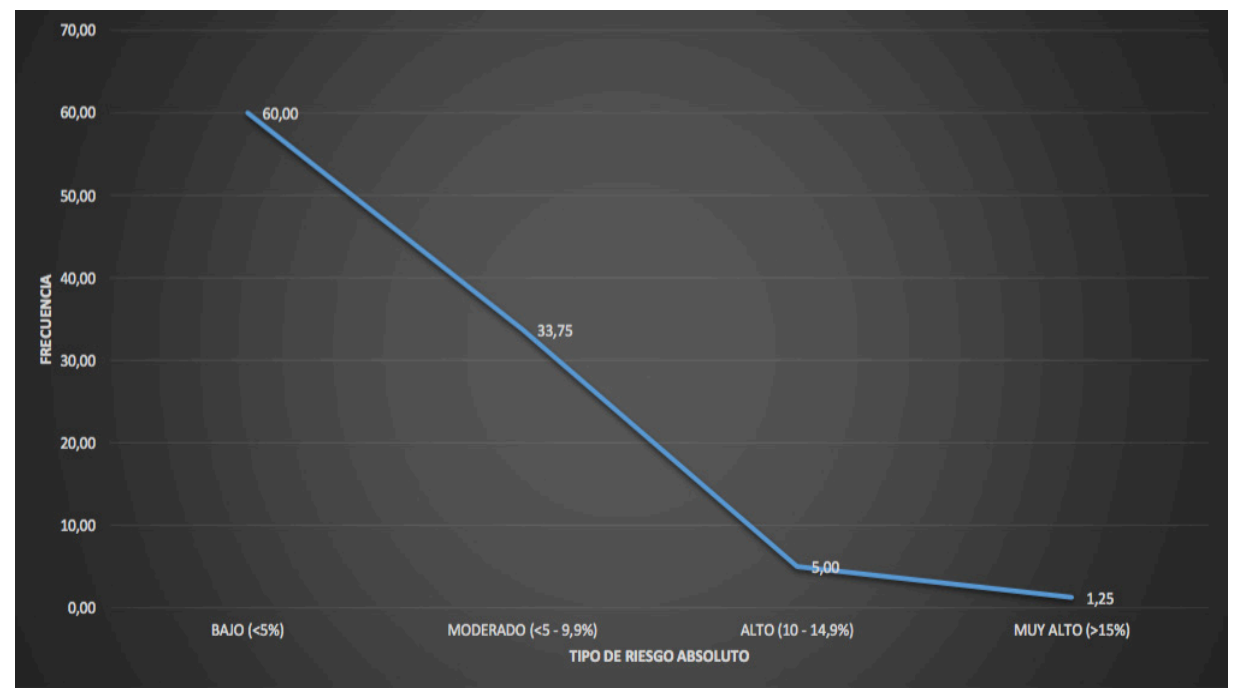

Figura 1. Riesgo cardiovascular absoluto

lo que la interpretación de estos factores de riesgos, a través del Score de Framingham, ayudará a identificar y disminuir la enfermedad cardiovascular.

Se ha llevado a cabo esta investigación tomando como base el cálculo del riesgo cardiovascular de la población militar a través del uso de las tablas de Framingham, las cuales han sido catalogadas como herramientas de alta fidelidad en la predicción del riesgo cardiovascular (Álvarez, 2001). Se ha evitado el uso de otras herramientas con gran uso en regiones de Europa, por su variabilidad en los resultados. No obstante, se debe ser prudentes con los resultados, ya que fueron realizados a través de una función de cálculo de riesgo obtenida de una población anglosajona. La adaptación de los datos se la realiza como una forma de adquirir información que permita tomar decisiones de prevención en la población militar.

Se observa que los factores de riesgo más importantes en la población militar están representados por HDL bajo en el $80 \%$, seguida de sobrepeso en el $46.25 \%$ y colesterol elevado en el $41.25 \%$. Los resultados obtenidos por Tovar et al. (2012), durante el período 2010 - 2011, en la población de aviadores del ejército colombiano, con una muestra de 1317 aviadores, fueron semejantes. En esa muestra, los principales factores de riesgo estuvieron representados por HDL bajo en el $71.07 \%$, seguido de sobrepeso en el $55.12 \%$ y colesterol elevado en el $53.53 \%$ (Tovar et al. 2012). Por otro lado, los resultados son diferentes a los de Arboleda y García, obtenidos durante el año 2016, en una población general de la región amazónica de Morona Santiago, con una muestra de 249 personas, en donde el tabaquismo (48.6\%) es el principal factor de riesgo, seguido del sobrepeso (40.2\%) y HDL bajo (10.4\%) (Arboleda y García 2017). Por tanto, el tabaquismo es el principal factor de riesgo en la población general.

El riesgo absoluto en la presente investigación es bajo en el $60 \%$ de la población, seguido de riesgo moderado en el $33.75 \%$, similar a los datos obtenidos por Auquilla, en el año 2010, en el personal militar de la Caballería Blindada $N^{\circ} 11$ Galápagos en Chimborazo (Ecuador), con una muestra de 300 participantes. Los resultados de dicha muestra determinaron que el riesgo bajo se representa en el $57 \%$ de la población y el riesgo moderado en el 29\% (Auquilla 2011). En contraste con los datos obtenidos en la investigación de Giraldo-Trujillo et al, realizada de la Universidad de Pereira (Colombia), con una muestra de 138 participantes, donde se mostró que el riesgo bajo estaba representado por el $28.6 \%$ y el riesgo moderado en el 53.5\% (Giraldo-Trujillo et al. 2011). Esto evidencia una diferencia en el porcentaje de riesgo entre la población militar y la población general.

El riesgo según la edad está acorde a lo esperado en la población entre 30 y 39 años con un $81.63 \%$ de riesgo bajo. En cuanto a la población de entre 40 y 49 años, se evidencia riesgo moderado en el $60 \%$ de los casos, en contraste con los resultados obtenidos por Cabrera, en el período 2010 - 2011, en los Combatientes del Este, La Habana (Cuba), con una muestra de 102 participantes, donde se evidenció un riesgo bajo en el $60.7 \%$, y riesgo moderado en el $14.7 \%$ en el mismo grupo etario (Cabrera y Suárez 2012). Estos son datos totalmente diferentes pero predecibles, ya que fueron obtenidos en una población cuyo sistema de salud se basa en la prevención. 


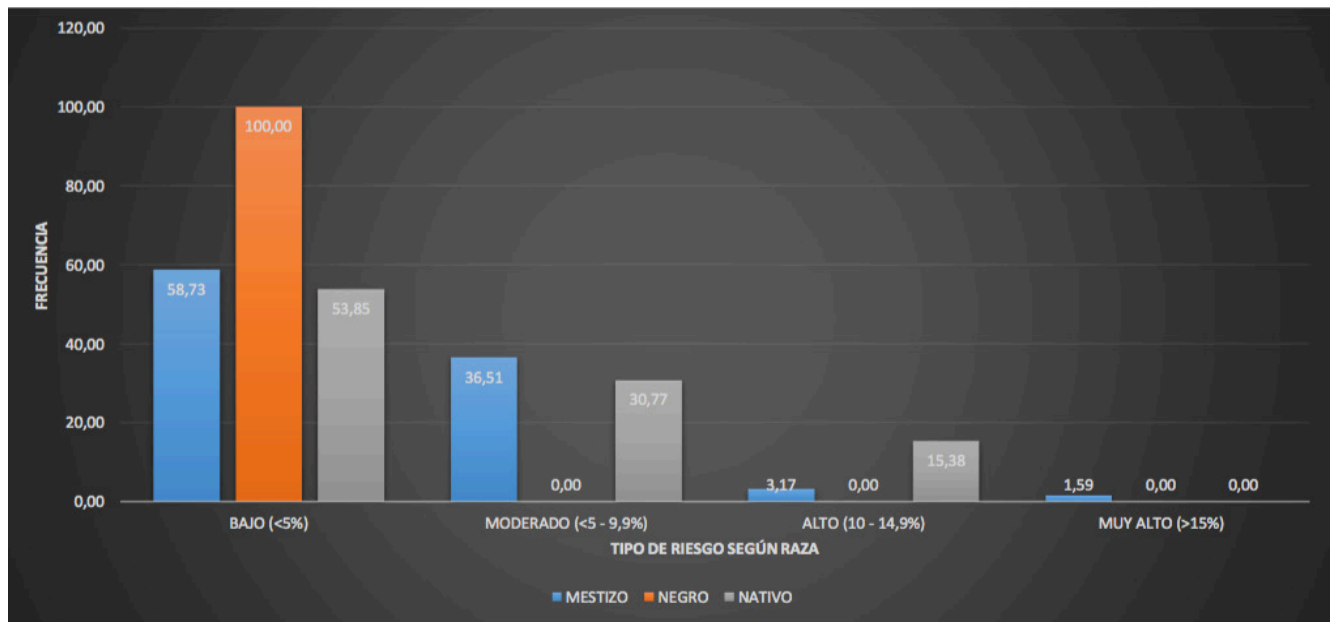

Figura 2. Riesgo cardiovascular según etnia

\section{CONCLUSIONES}

De acuerdo a los resultados, se establece que el personal militar de la población en estudio:

- $\quad$ Presenta un perfil epidemiológico similar a otras poblaciones militares en cuanto a los factores de riesgo y de riesgo total. El riesgo cardiovascular se presenta entre bajo y moderado, y tiene como principales factores de riesgo al HDL disminuido, al sobrepeso y al colesterol elevado.
- Se evidencia, además, una diferencia significativa entre la población general y el personal militar, en cuanto al riesgo cardiovascular y los principales factores de riesgo. El principal factor de riesgo en la población general se presenta por el tabaquismo, mismo que, al ser modificable, puede ser usado como estrategia para la disminución del riesgo total.

\section{AGRADECIMIENTOS}

Se agradece a las autoridades y al personal del Batallón de Selva No 62 “Zamora”, cuya predisposición permitió la realización de la presente investigación.

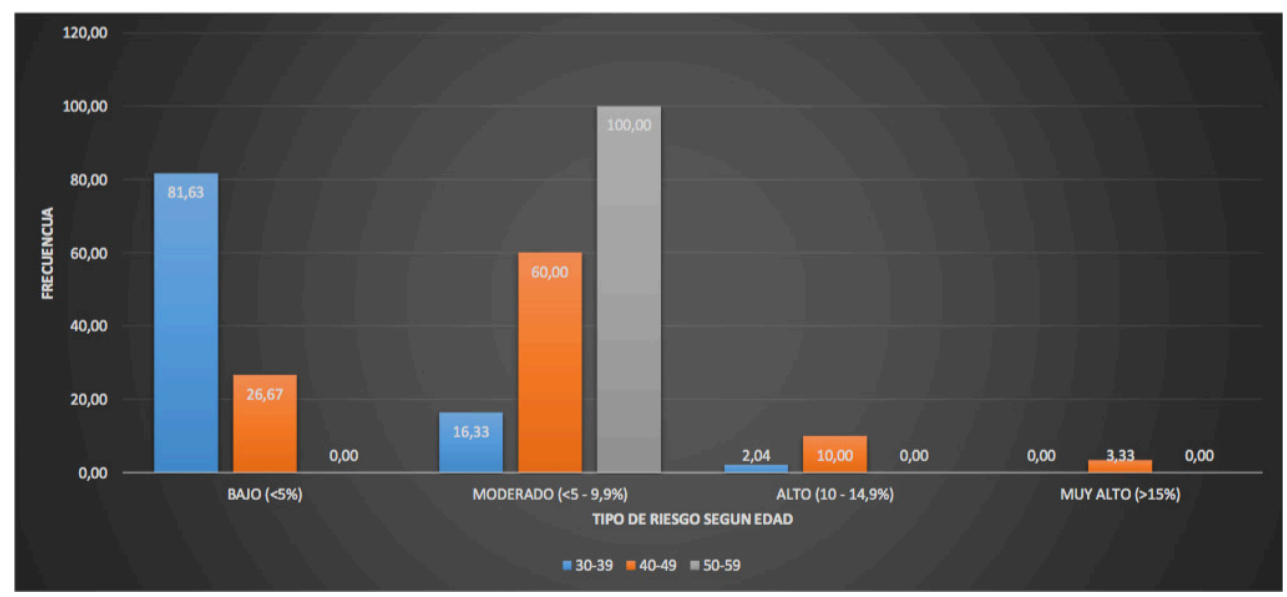

Figura 3. Riesgo cardiovascular según edad 


\section{REFERENCIAS BIBLIOGRÁFICAS}

[ALAD] Asociación Latinoamericana de Diabetes. 2013. Guía ALAD 2013 sobre el diagnóstico, control y tratamiendo de la diabetes mellitus tipo 2 con medicina basada en evidencia. Rev Asoc Latin de Diabet.

Alcocer L, Lozada O, Fanghanel G, Sánchez L, Campos E. 2011. Estratificación del riesgo cardiovascular global. comparaciónde los métodos Framingham y SCORE en población mexicana del estudio PRIT. Rev Cir Ciruj. 79: 168-74.

Álvarez A. 2001. Las tablas de riesgo cardiovascular. Una revisión crítica. MEDIFAM. 11(3): 122-39.

Amariles P, Machuca M, Jiménez A, Silva M, Sabater D, Baena M, Jimenez J, Faus M. 2004. Riesgo cardiovascular: componentes, valoración e intervenciones preventivas. Ars Pharm. [Internet]. [Date cited: 15/08/2017]. 45 (3): 187-210. Available from: http://farmacia.ugr.es/ars/pdf/290.pdf

Arboleda M, García A. 2017. Riesgo cardiovascular: analisis basado en las tablas de Framingham en pacientes asistidos en la unidad ambulatoria 309, IESS - Sucúa. Scielo. 25 (1): 20-30.

Armario P, Banegas J, Campo C, De la Sierra A, Gorostidi M, Hernández R. 2005. Guía española de hipertensión arterial.

Auquilla A. 2011. Determinación y prevalencia de factores de riesgo cardiovascular en el personal de la Brigada de Caballería Blindada No11 Galápagos, Riobamba 2010. Escuela Superior Politécnica de Chimborazo.

Backer G, De Ambrosioni E, Borch K, Brotons C, Cifkova R, Dallongeville J. 2003. European guidelines on cardiovascular disease prevention in clinical practice. Third Joint Task Force of European and other societies on cardiovascular disease prevention in clinical practice. Euro Heart J. 24: 1601-10.

Cabrera A, Alemán J, Rodríguez M, Del Castillo J, Domínguez S, Almeida D, Lafuente B, Aguirre A, Brito B. 2009. En la población canaria, la función de Framingham estima mejor el riesgo de mortalidad cardiovascular que la función SCORE. Gac Sanit. 23 (3): 216-21.

Cabrera S, Suárez R. 2012. Riesgo cardiovascular global en una población de combatientes. Rev Haban Cienc Méd. 11 (3). D'Agostino R, Vasan R, Pencina M, Wolf P, Cobain M, Massaro J, Kannel, W. 2008. General cardiovascular risk profile for use in primary care - the Framingham Heart Study. Circulation - AHA, [Internet]. [Date cited: 15/08/2017]. 117: 743-53. Available from: http://circ.ahajournals.org/content/117/6/743. full\#ref-list-1

Dawber T, Meadors G, Moore F. 1951. Epidemiological approaches to heart disease: The Framingham Study. $\mathrm{Am} J \mathrm{Pu}$ - blic Health. 41: 279-86.

Dawber T, Moore F, Mann G. 1957. Coronary heart disease in the Framingham Study. Am J Public Health. 47: 4-24.

[FHS] Framingham Heart Study. 2016. Cardiovascular disease (10-Year Risk). [Internet]. [Date cited: 15/08/2017]. Available from: https://www.framinghamheartstudy.org/risk-functions/ cardiovascular-disease/general-cvd-risk-prediction-using-bmi. php

Galán A, García F, Taix M, Ros X, Martí A, Gil A. 2004. Riesgo cardiovascular del SCORE comparado con el de Framingham. Consecuencias del cambio propuesto por las sociedades Europeas. Med Clin. 123 (18): 681-85.

Giraldo-Trujillo J, Martinez J, Granada-Echeverry P. 2011. Aplicación de la escala de Framingham en la detección de riesgo cardiovascular en empleados universitarios, 2008. Rev Salud Publica. [Internet]. [Date cited: 15/08/2017]. 13 (4): 633-43. Available from: https://www.scielosp.org/article/ssm/ content/raw/?resource_ssm_path=/media/assets/rsap/v13n4/ v13n4a08.pdf

González C, Rodilla E, Costa J, Justicia J, Pascual J. 2006. Comparación entre el algoritmo de Framingham Y el de SCO$\mathrm{RE}$ en el cálculo del riesgo cardiovascular en sujetos de 40-65 Años. Rev Med Clin. 126 (14): 527-31.

Icaza G, Nuñez L, Marrugat J, Mujica V, Escobar C, Jiménez A, Pérez, P, Palomo I. 2009. Estimación de riesgo de enfermedad coronaria mediante la función de Framingham adaptada para la población chilena. Rev Med Chil. 137 (10): 1273-82

Lobos J, Castellanos A. 2006. Factores de riesgo cardiovasculares. J\&C Edic Med. [Internet]. [Date cited: 15/08/2017]. 3: 107-14. Available from: http://mail.jc-edicionesmedicas.com/ en/system/files/documentos/p107_114_Factores_riesgo.pdf

Lobos J, Brotons C. 2011. Factores de riesgo cardiovascular y atención primaria: evaluación e intervención. Elsevier. [Internet]. [Date cited: 15/08/2017]. 43 (12). Available from: http:// www.elsevier.es/es-revista-atencion-primaria-27-articulo-factores-riesgo-cardiovascular-atencion-primaria-90055363

Jaume M, Solanas P, D’Agostino R, Sullivan L, Ordovas J, Cordón F, Ramos R. 2003. Estimación del riesgo coronario en España mediante la ecuación de Framingham calibrada. Rev Esp Card. 56 (3): 253-61.

Mostaza J, Vicente I, Taboada M, Laguna F, Echániz A, García F, Lahoz C. 2005. La aplicación de las tablas del SCORE a varones de edad avanzada triplica el número de sujetos clasificados de alto riesgo en comparación con la función Framingham. Med Clin. 124 (13): 487-90. 
Muñoz O, Rodríguez N, Ruiz A, Rondón M. 2014. Validación de los modelos de predicción de Framingham Y PROCAM como estimadores del riesgo cardiovasculares en una población colombiana. Rev Colom Card. 21 (4): 202-12.

[NHI] National Heart Institution. 1957. A simposium: measuring the risk of coronary heart disease in adult population groups. Am J Public Health. 47:1-63.

O’Donell C, Elousa R. 2008. Factores de riesgo cardiovasculares. perspectivas derivadas del Framingham Hearth Study. Rev Esp Card. 61 (3): 299-310.

[OMS] Organización Mundial de Salud. 2015. Enfermedades cardiovasculares. Centro de Prensa. [Internet]. [Date cited: 15/08/2017]. Available from: http://www.who.int/mediacentre/ factsheets/fs317/es/

Splansky G, Corey D, Yang Q, Arwood L, Cupples L, Benja$\min$ E. 2007. The third generation cohort of the National Lung, and Blood Intitute's Framingham Heart Study: design, recruitment, and initial examination. Am J Epidemiol. 165: 1328-35.

Tovar R, Daza M, Godoy G, Herrera Y, Sánchez L. 2012. Evaluación del riesgo cardiovascular usando la escala de FRAMINGHAM en aviadores militares del ejército colombiano." Universidad del Rosario.

Vega J, Guimará M, Vega L. 2011. Riesgo cardiovascular, una herramienta útil para la prevención de las enfermedades cardiovasculares. Rev Cubana de Med Gen Integr. 27 (1). 